\title{
Understanding DÉJÀ VU
}

\section{Kisa Fatima Altaf}

Advance Educational Institute and Research Centre

Dadabhoy Institute of Higher Education

Corresponding Author: Kisa@aeirc-edu.com

Have you ever witnessed that, say for instance, you are migrating to Northern areas of Pakistan for the first time or you are playing basketball on a court and being cheered up by audience shouting only your name, and all of a sudden it feels like as if you have been in that very moment before or maybe you are having pizza with a group of friends, teasing a friend, and you have the feeling that you've already experienced this very thing -- same friends, same dinner, and same topic $60 \%$ of the populations, those who have experienced it for the first time assume it as Reincarnation.

The phenomenon is comparatively complicated, and termed as Déjà vu. The word déjà vu is French and means, actual, "seen before". Those who have witnessed the feeling defined it as an astounding sense of familiarity with something that shouldn't be usual at all.

There are various logics on that. Absolute reason is, sometimes our instinct gets puzzled that the occasion is happening in the present or past. So for 1-2 seconds, we sense like we have lived this minute before. But not actually, $67 \%$ of people have had a déjà vu experience (a median of 41 surveys).

Charles Dickens mentioned that We all have some wisdom of an awareness, that comes over us sometimes, of what we are doing and saying having been said and done before, in an isolated time - of our having been surrounded, dim ages ago, by the same faces, substances, and prospects - of our knowing completely what will be said next, as if we suddenly remembered it!

There are countless philosophies as to why déjà vu happens. Swiss scholar Arthur Funkhouser proposes that there are certain "déjà experiences" and defends that in order to study better the phenomenon, the implication among the experiences need to be recognized. In the examples quoted above, Funkhouser would describe the first incidence as déjà visite ("visited already") and the other as déjà vecu/ déjà vu ("seen before or lived through").
Deja Vu refers to those different and generally strange moments when the current moment feels like the former. It is difficult to explain. A few people hunt their thoughts for dreams that might have been like the current situation. Others think that the action is what appears when things from past lives proceeds in this one. Both assumptions are impractical to justify, confound, or (until recently), interrogate. The assumption that it is about previous lives is a matter of belief. The perception that it has to do with dreams is less a matter of belief - only a specified people plea to recall previous lives, but relatively everyone commemorates few of their dreams. Some recall many of them. The concept of reincarnation that is most persistent with modernized brain science concludes that no thoughts or memories are passed from one life to the other. What is shifted is a set of alarms that emulates the states of consciousness. Thoughts don't need to go along. (Todd Murphy 1999)

As I know and can judge, dream is a fantasy or projection of visuals we see every day but with different scenarios we have never been through. It is a process in which the physiological aspects of the body and its psychological responses by the brain are saved in a part of the brain called sub-conscious. It is a part of the mind where the memories are saved and are highly approachable in dreams as all the other functions of the body are hibernated during sleep. (W. Hassan 2014). The subconscious and conscious mind is intertwined involving the same point in space and time at any one moment. The most persistent data in the déjà vu history is that the prevalence with which it is experienced declines with age. As well as $75 \%$ people reports that they have experienced few forms of déjà vu. A large number of incidents happen in people of about 15 to 25 years older than in any other age group.

Déjà-vu is a non-cognitive fault in which a situation has the sense of intimacy despite there being no definite antecedent in the past. Incidence of déjà-vu is most when people are in their 20s and almost fade after age 50. (Brown, Alan S. 2003). 
Since déjà vu happens in people with and without any medical status, there is much belief as to how and why this episode happens. Many psychoanalysts characterized déjà vu to simple illusion or gratification; at the same time some psychiatrists attribute it to an imbalance in the brain that originates the brain to oversight the present for the past.

Some interesting researches on brain function cites that what we concern as "deja vu" is basically a "misfire" of the brain in between notion and encoding into memory, by which something that we "see" is literally encoded twice -- once, subliminally, and the next superluminally. This left us with the "feeling" that we have experienced what we are observing in the past, because in our brains, we have. There is a massive time lag between all of our actions happening at the certain moment which is being composed at the subconscious level and the conscious point we experience as the real moment.

\section{Conclusion}

Many parapsychologists consider that it is linked to a past-life experience. Certainly, there are more researches, studies and reviews to be done. Time only runs in one direction.

What I conclude is that deja vu is a sensual paradox, and not associated to time itself. Neuroscientists have come up with many assumptions about the experience of déjà vu, but no one had ever been justified. Authors and scientists have presented mystical statements.

\section{Reference}

- Brown, Alan S. "A review of the deja vu experience." Psychological bulletin 129.3 (2003): 394.

- W.Hassan (2014). Understanding Dreams. Annals of Psychophysology (1) 14-15.

- Todd Murphy (1999). “The experience of déjà vu in clinical and spiritual terms." Sacred Pathway: The brains role in Religious and Mystic Experiences. 